\title{
Rheological Link Between Polymer Melts with a High Molecular Weight Tail and Enhanced Formation of Shish-Kebabs
}

Wingstrand, Sara Lindeblad; Shen, Bo; Kornfield, Julie A.; Mortensen, Kell; Parisi, Daniele; Vlassopoulos, Dimitris; Hassager, Ole

Published in:

A C S Macro Letters

Link to article, DOI:

10.1021/acsmacrolett.7b00718

Publication date:

2017

Document Version

Peer reviewed version

Link back to DTU Orbit

Citation (APA):

Wingstrand, S. L., Shen, B., Kornfield, J. A., Mortensen, K., Parisi, D., Vlassopoulos, D., \& Hassager, O. (2017). Rheological Link Between Polymer Melts with a High Molecular Weight Tail and Enhanced Formation of ShishKebabs. A C S Macro Letters, 6, 1268-1273. https://doi.org/10.1021/acsmacrolett.7b00718

\section{General rights}

Copyright and moral rights for the publications made accessible in the public portal are retained by the authors and/or other copyright owners and it is a condition of accessing publications that users recognise and abide by the legal requirements associated with these rights.

- Users may download and print one copy of any publication from the public portal for the purpose of private study or research.

- You may not further distribute the material or use it for any profit-making activity or commercial gain

- You may freely distribute the URL identifying the publication in the public portal 


\title{
Rheological Link Between Polymer Melts with
}

\section{a High Molecular Weight Tail and Enhanced Formation of Shish-kebabs}

Sara Lindeblad Wingstrand, ${ }^{\dagger}$ Bo Shen, ${ }^{\ddagger}$ Julie A. Kornfield, ${ }^{\ddagger}$ Kell Mortensen,, Daniele Parisi, ${ }^{\S} \|$ Dimitris Vlassopoulos, ${ }^{\S}, \|$ and Ole Hassager ${ }^{*, \dagger}$

$\dagger$ Technical University of Denmark, Department of Chemical and Biochemcial Engineering, Danish Polymer Center,DK-2800 Kgs. Lyngby, Denmark

$\ddagger$ California Institute of Technology, Division of Chemistry and Chemical Engineering, Pasadena, CA 91125, USA

【University of Copenhagen, Niels Bohr Institute, X-ray and Neutron Science, DK-2100 København Ø, Denmark

§Institute of Electronic Structure and Laser, FORTH, Heraklion 71110, Crete Greece || Department of Materials Science \& Technology, University of Crete, Heraklion 71003, Crete Greece

E-mail: oh@kt.dtu.dk

\begin{abstract}
Presence of an ultra high molecular weight (UHMw) fraction in flowing polymer melts, is known to facilitate formation of oriented crystalline structures significantly. The UHMw fraction manifests itself as a minor tail in the molar mass distribution and is hardly detectable in the canonical characterization methods. In this study, alternatively, we demonstrate how the nonlinear extensional rheology reveals to be a very
\end{abstract}


sensitive characterization tool for investigating the effect of the UHMw-tail on the structural ordering mechanism. Samples containing an UHMw-tail relative to samples without, exhibit a clear increase in extensional stress that is directly correlated with the crystalline orientation of the quenched samples. Extensional rheology, particularly, in combination with linear creep measurements, thus enables the conformational evolution of the UHMw-tail to be studied and linked to the enhanced formation of oriented structures.

The morphology of semicrystalline polymeric products varies greatly depending on the imposed flow conditions during melt processing and on the molecular chain architecture. ${ }^{1,2}$ The combination of flow and chain architecture determines the degree of chain stretch and hence the structure into which, the polymer crystallizes. ${ }^{3,4}$ The morphology is important for the mechanical properties of the final product. Especially the creation of oriented crystal structures (shish-kebabs) rather than isotropic structures (spherulites) is key to enhancement of directional mechanical strength. ${ }^{5-8}$

One way to obtain shish-kebabs from a molten, relaxed polymer melt is by application of flow. A sufficiently strong flow will result in stretching of the chains. ${ }^{9,10}$ Under the right thermal conditions, the stretched chains form rodlike nuclei that grows into long fibrils (shish) on which subsequent overgrowth of oriented lamellae (kebabs) perpendicular to the shish occurs ${ }^{11-13}$.

Formation of shish-kebabs is enhanced by the presence of a small fraction of chains having a molar mass considerably higher than the rest of the melt - " an ultra high molecular weight tail (UHMw-tail)". ${ }^{14-21}$ Even when the concentration of UHMw chains is so low that they hardly affect most other properties of the melt, such as the viscosity measured in the conventionally used frequency range, thermal properties and quiescent crystallization kinetics, they significantly enhance the formation of shish-kebabs. ${ }^{14}$

One property that has not been investigated for semicrystalline polymers containing an nearly invisible high molecular weight tail is their response in controlled uniaxial extensional 
flow. Inherently, extensional flows are considered strong flows whereby their ability to stretch the polymer molecules is much greater than shear flow. ${ }^{22,23}$ Furthermore the extensional response of the liquid is extremely sensitive to stretching of the chains. Due to experimental difficulties, the number of studies connecting crystallization and rheology in extension is quite limited. ${ }^{24-31}$ Studies on semicrystalline systems of known molecular composition are still to be studied in controlled uniaxial extension.

In this work we investigate the role of the high molecular weight tail in controlled uniaxial extension as well as the morphology obtained after quenching of the melt. We compare a blend of $1 \%$ UHMw polyethylene $(\mathrm{UHMwPE})\left(M_{w}=4000 \mathrm{~kg} / \mathrm{mol}\right)$ in a matrix of commercial HDPE - high density polyethylene (632-D1 from Dow, $M_{w}=156 \mathrm{~kg} / \mathrm{mol}$ ) - with the pure matrix, referred to as the "UH-blend" and the "matrix", respectively. The molecular weight

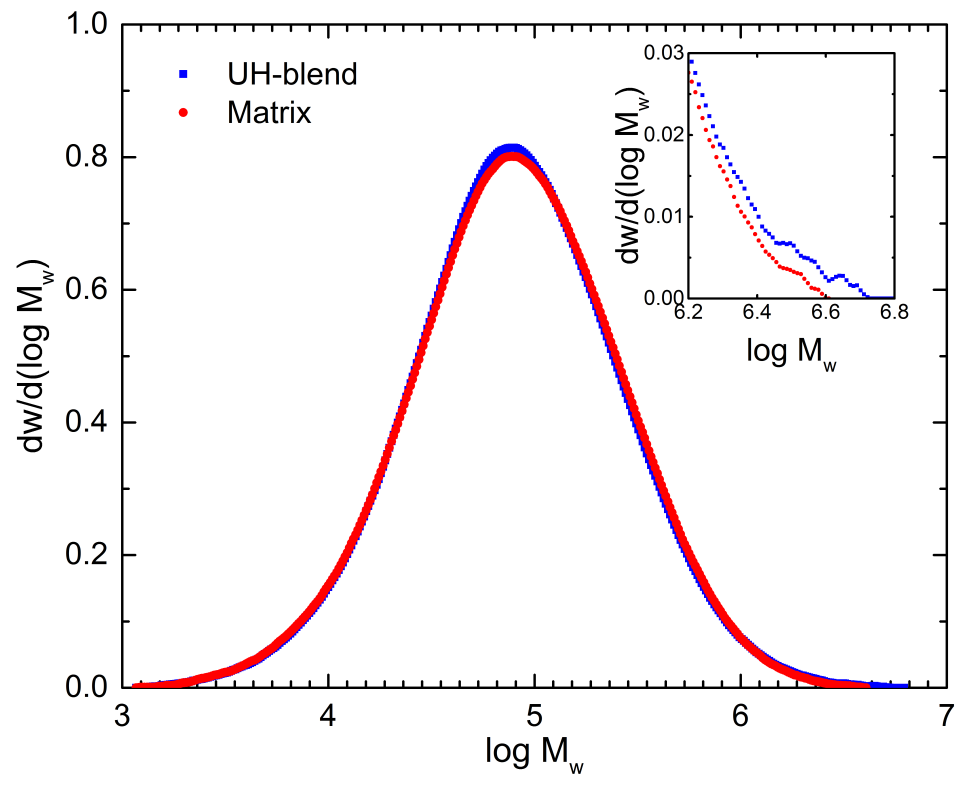

Figure 1: Molecular weight distribution obtained via GT-GPC (detection by refractive index) of the UH-blend (blue) and the matrix (red). The inset shows a magnification of the UHMwfraction.

distribution (MWD) (Figure 1), number, weight and z-average molar masses $\left(M_{n}, M_{w}\right.$ and $M_{z}$ respectively) as well as $T_{m}$ (see Table 1) are nearly identical for the two samples. We study the rheological behavior in the linear regime using small-amplitude oscillatory shear (SAOS), at temperatures between 140 and $190^{\circ} \mathrm{C}$ in combination with creep at $150^{\circ} \mathrm{C}$. The 
creep compliance data is converted into dynamic compliance and then to dynamic moduli by Fourier transformations. In this way we obtained the linear viscoelastic response over a broad range of frequencies at a reference temperature of $140{ }^{\circ} \mathrm{C}$. The nonlinear behvior is studied in controlled uniaxial extension at $140^{\circ} \mathrm{C}$. Here we measure the extensional stress $\sigma_{z z}-\sigma_{r r}$ during deformations of constant Hencky strain rates $\dot{\varepsilon}$. All samples are quenched to room temperature at a Hencky strain of $\varepsilon=5.5$ and small angle X-ray scattering (SAXS) patterns for the quenched samples, are collected ex-situ.

Table 1: Material characteristics for the UH-blend and the matrix. solutions.

\begin{tabular}{|l|c|c|c|c|c|}
\hline \hline Sample & $\phi(\mathrm{w}$ frac $)$ & $M_{n}(\mathrm{~kg} / \mathrm{mol})$ & $M_{w}(\mathrm{~kg} / \mathrm{mol})$ & $M_{z}(\mathrm{~kg} / \mathrm{mol})$ & $T_{m}\left({ }^{\circ} \mathrm{C}\right)$ \\
\hline Matrix & - & 40 & 156 & 495 & $138.4 \pm 0.5$ \\
\hline UH-blend & 0.01 & 40 & 156 & 550 & $138.9 \pm 0.5$ \\
\hline
\end{tabular}

We use Herman's orientation factor $F_{H}$ extracted from SAXS patterns as a measure for the average degree of crystalline orientation in the quenched samples. $F_{H}$ is given by the second Legendre polynomial:

$$
F_{H}=\frac{3\left\langle\cos ^{2} \phi\right\rangle-1}{2}
$$

Where $\left\langle\cos ^{2} \phi\right\rangle$ is the average cosine squared of the angle $\phi$ given as the angle between the normal of a plane in the crystalline domains and a given macroscopic direction. We determine $F_{H}$ from SAXS patterns using scattering between kebabs growing perpendicular to the shish aligned along the flow direction. $\phi$ is defined as the angle between the normal of the kebab-planes and the macroscopic direction set to be the stretching direction ${ }^{1}$ (see Supporting Information).

Figure 2 shows the SAXS patterns along with Herman's orientation factor versus the Hencky strain rate during stretching. While both samples show a systematic increase with extension rate, the orientation factor for the UH-blend is clearly higher relative to the matrix especially at high rates. The enhanced formation of oriented structures upon addition of just small amounts of UHMw-component is thus confirmed for extensional flows, which is in agreement with previous studies showing same effect for shear flows. ${ }^{14}$ 


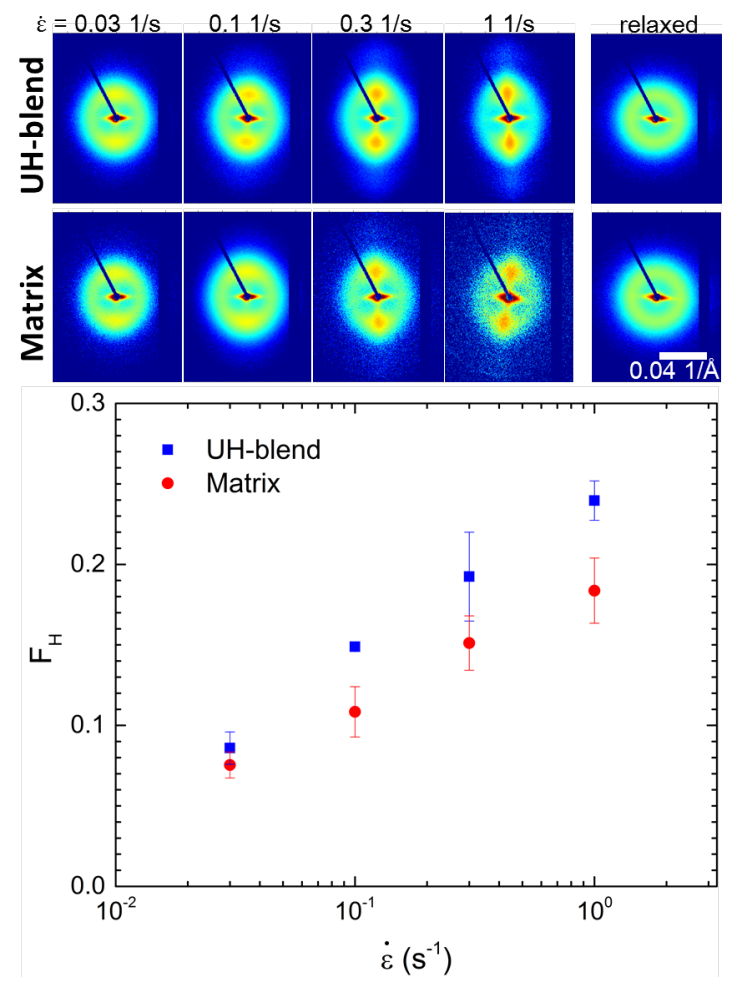

Figure 2: Oritentation of quenched filaments. Top: SAXS patterns (vertical stretching direction). Bottom: Herman's orientation factor for the UH-blend (blue symbols) and the matrix (red symbols). 
The linear and nonlinear extensional rheological response of the two samples are shown in Figures 3a and b, respectively. The linear responses (Figure 3a) are modelled using a multi mode Maxwell spectrum, a discrete spectrum of relaxation times $\tau_{i}$ with corresponding moduli $g_{i}$ (see Supporting Information). Both data and corresponding fits for the two samples are identical in the high frequency region that corresponds to relaxation on length scales shorter than the entire length of the UHMw-tail. A significant difference in behavior is detected only in the region just before entering the terminal regime, i.e., the regime where $G^{\prime}$ and $G^{\prime \prime}$ reach slopes of 2 and 1 , respectively. The addition of $1 \mathrm{w} \%$ UHMwPE is responsible for the formation of a shoulder in $G^{\prime}$, indicative of an additional mode which slows-down the terminal relaxation substantially (see also the difference in zero-shear-rate viscosity in Supporting Information Figure 5S). Indeed, for the 10th Maxwell mode $\left(\tau_{10}=10^{3} \mathrm{~s}\right.$ see inset in Figure 3a) the difference in $g_{10}$ between the two samples is a factor of $\sim 10^{3}$. It turns out that the 10th mode of the matrix can be left out without compromising the Maxwell fit, while it is essential for the fit of the UH-blend. For the linear data obtained from SAOS, it is important to note that due to the rapidly decreasing torque signal upon lowering the frequency, the lower instrumental limit is reached around a frequency $\omega=0.01 \mathrm{rad} / \mathrm{s}$. Thus, the spectrum obtainable from SAOS (i.e. data contained in the grey square) suggests that the two samples are identical, just like the MWD and $T_{m}$ suggested. Only with the additional creep data, can any significant difference be detected as pointed out by Münstedt and co-workers. ${ }^{32,33}$

The nonlinear extensional response (Figure 3b), reported in terms of the extensional stress growth coefficient $\eta_{E}^{+}=\left(\sigma_{z z}-\sigma_{r r}\right) / \dot{\varepsilon}$ shows a clear difference between the two samples. Although both samples strain harden, the extent of strain hardening is much larger for UHblend relative to the matrix. Initially all samples follow the LVE prediction and overlap at small $t$ (Figure $3 \mathrm{~b}$ inset). Prior to quench at $\varepsilon=5.5$, all samples reach steady elongational flow which is confirmed by complementary nonlinear uniaxial creep measurements showing same steady elongational viscosity ${ }^{34}$ (see Figure S6 in Supporting Information). 


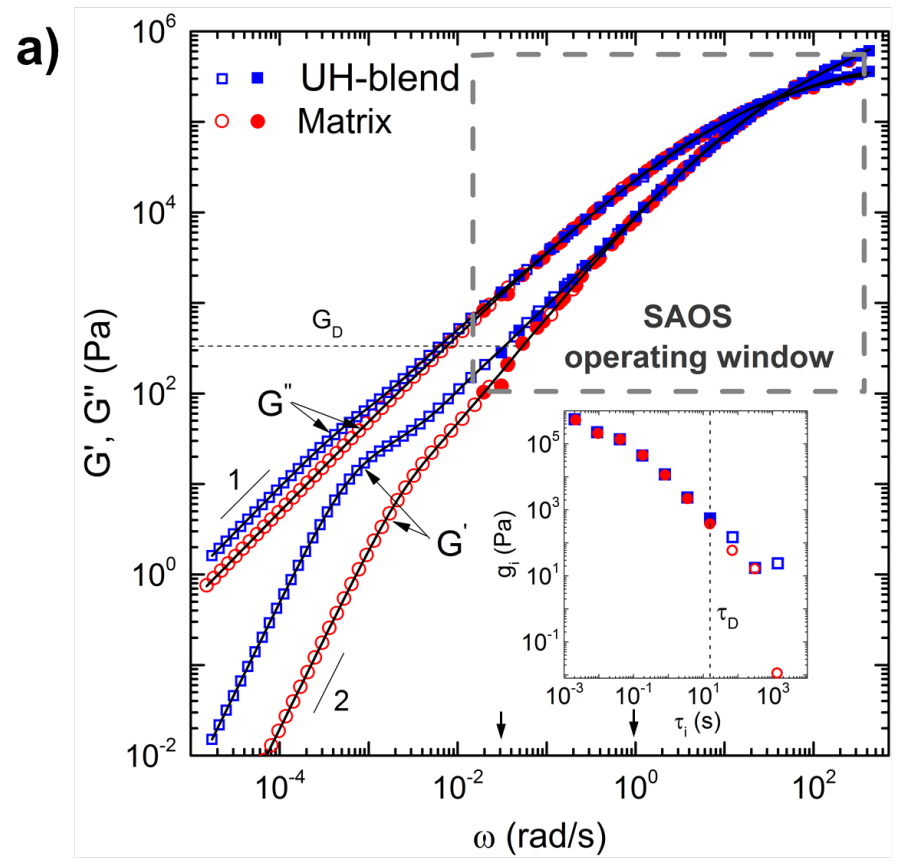

b)

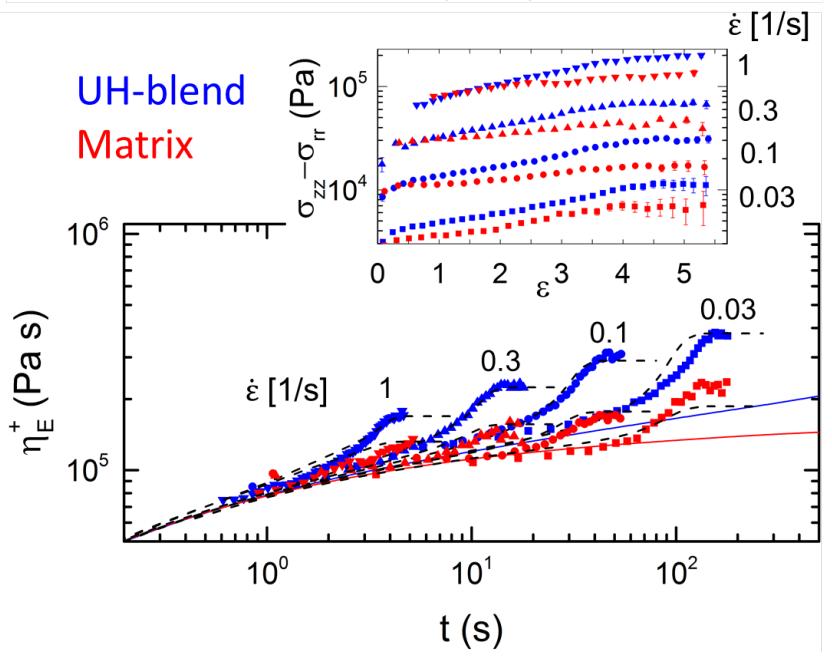

Figure 3: Rheology of the UH-blend (blue symbols) and the matrix (red symbols) at $140^{\circ} \mathrm{C}$. (a) Linear response, storage $\left(G^{\prime}\right)$ and loss modulus $\left(G^{\prime \prime}\right)$, obtained via SAOS (closed symbols) and via creep (open symbols). Solid lines show the prediction by the multimode Maxwell model (10 modes). Arrows on the x-axis indicate the range of $\dot{\varepsilon}$ in extension. Inset: Maxwell moduli $g_{i}$ versus time constants $\tau_{i}$. (b) Nonlinear extensional response in terms of extensional stress growth coefficient $\eta_{E}^{+}$together with the linear viscoelastic envelope (blue and red lineas) and HMMSF model-prediction (black dashed lines) using the nonlinear fitting parameter $G_{D}=350 \mathrm{~Pa}$ (see text). Inset: extensional stress versus Hencky strain. 
The extensional response is modelled using the hierarchical multimode molecular stress function (HMMSF) as it is able to properly capture the transient and steady state response of a polydisperse polymer melt using only one nonlinear fit parameter $G_{D} \cdot{ }^{35}$ The model considers the polydisperse samples as a collection of chain segments of various relaxation times obtained from the multimode Maxwell model. ${ }^{36}$ Once a segment is relaxed it acts as a solvent for the remaining unrelaxed chains. The nonlinear parameter $G_{D}$ is a point along the relaxation modulus $G(t)$ that separates permanently diluted segments from dynamically diluted segments. $G_{D}$ is associated with a time constant $\tau_{D}$ defined by $G_{D}=G\left(\tau_{D}\right)$ for the onset of dynamic dilution. The extra stress tensor, calculated as a sum of stress contributions from each Maxwell mode, is given by:

$$
\boldsymbol{\sigma}=\sum_{i} \int_{-\infty}^{t} \frac{\partial G_{i}\left(t-t^{\prime}\right)}{\partial t^{\prime}} \mathbf{S}_{\mathbf{D E}}^{\mathbf{I A}}\left(t, t^{\prime}\right) f_{i}\left(t, t^{\prime}\right)^{2} d t^{\prime}
$$

Here $G_{i}\left(t-t^{\prime}\right)=g_{i} \exp \left(\frac{t-t^{\prime}}{\tau_{i}}\right)$ is the relaxation modulus and $f_{i}$ is the segmental chain stretch of mode $i{ }^{35} \mathrm{~S}_{\mathrm{DE}}^{\mathrm{IA}}$ is the Doi-Edwards orientation tensor with the independent alignment assumption. ${ }^{37-39}$ Both $\mathbf{S}_{\mathrm{DE}}^{\mathrm{IA}}$ and $f_{i}$ are functions of the observation time $t$ and the time $t^{\prime}$ at which new tube segments are created by reptation. Consequently, the middle segments of the chains will be more stretched and oriented compared with the end segments that relax much faster. The evolution of $\mathrm{S}_{\mathrm{DE}}^{\mathrm{IA}}$ can conveniently be described by the a product of the Finger strain tensor $\mathbf{B}$ and a damping function $h(\varepsilon)$ :

$$
\mathbf{S}_{\mathbf{D E}}^{\mathbf{I A}}\left(t, t^{\prime}\right)=h\left(t, t^{\prime}\right) \mathbf{B}(t, t)
$$

We use the analytic expression of $h\left(t, t^{\prime}\right)$ as found in Urakawa et al., ${ }^{40}$ the definition of $\mathbf{B}$ given by Bird et al., ${ }^{22}$ and $f_{i}$ defined as the inverse relative tube diameter $f_{i}\left(t, t^{\prime}\right)=$ $a_{0} / a_{i}\left(t, t^{\prime}\right)$. The evolution of $f_{i}$ is given by a convective term and two relaxation terms:

$$
\left.\frac{\partial f_{i}}{\partial t}=f_{i}(\boldsymbol{\kappa}: \mathbf{S})\right)-3 \frac{f_{i}-1}{\tau_{i}}\left(1-\frac{2}{3} w_{i}^{2}\right)-\frac{2}{3} \frac{f_{i}^{2}\left(f_{i}^{3}-1\right)}{\tau_{i}} w_{i}^{2}
$$


Here $\boldsymbol{\kappa}$ is the velocity gradient tensor. The parameter $w_{i}$ is the fraction of dynamically diluted chain segments of mode $\mathrm{i}$, which is only less than 1 for modes that have $\tau_{i}>\tau_{D}$. The chain segments that have $\tau_{i}<\tau_{D}$ are considered as permanently diluted.

$$
\begin{array}{r}
w_{i}^{2}=\frac{1}{G_{D}} \sum_{j} g_{j} \exp \left(-\frac{\tau_{i}}{\tau_{j}}\right) \quad \text { for } \quad \tau_{i}>\tau_{D} \\
w_{i}^{2}=1 \quad \text { for } \quad \tau_{i}<\tau_{D}
\end{array}
$$

The HMMSF predictions, shown in Figure 3b, have been obtained with the nonlinear parameter $G_{D}=350 \mathrm{~Pa}$ adjusted to describe the steady extensional stress values. In addition, the success of the fit is crucially dependent upon the application of the complete low frequency response in the linear regime. The combination of creep and controlled extension thereby enables the evolution of the stretch for each mode to be studied in detail. The HMMSF modelling reveals that the difference does indeed arise from the very large stretch of the highest modes that furthermore have a squared contribution to stress. As a result the stress contribution at $\dot{\varepsilon}=0.1 \mathrm{~s}^{-1}$ from the longest mode is $53 \mathrm{~Pa}$ for the matrix while it is 17610 Pa for the UH-blend (see Figure 4a).

In trying to explain why the presence of a UHMw-tail increases the potential for oriented structures we utilize relations connecting chain stretch and nucleation rates in combination with the HMMSF model. Peters and co-workers have found that the rate of shish nucleation $\dot{N}_{s}$ in a polymer melt containing a small UHMw-fraction has a power law dependency on the stretch $\Lambda_{i}$ of the UHMw-component. ${ }^{41-43}$ Specifically they find that $\dot{N}_{s} \propto \Lambda^{4}-1$ at the onset of crystallization. Based on ideas by the Rolie-Poly model they define $\Lambda_{i}$ from the stress tensor expression non-dimensionalized by the modulus. ${ }^{44}$ In the following we therefore associate the relevant stretch ratio with the segmental stretch $f_{i}$ as defined above. In the present study where the molar masses of the matrix and the blend are not completely separated, it is not solely the UHMw-component that is stretching, but the highest molar mass fraction of the matrix as well. To account for that, we calculate an average crystalliza- 

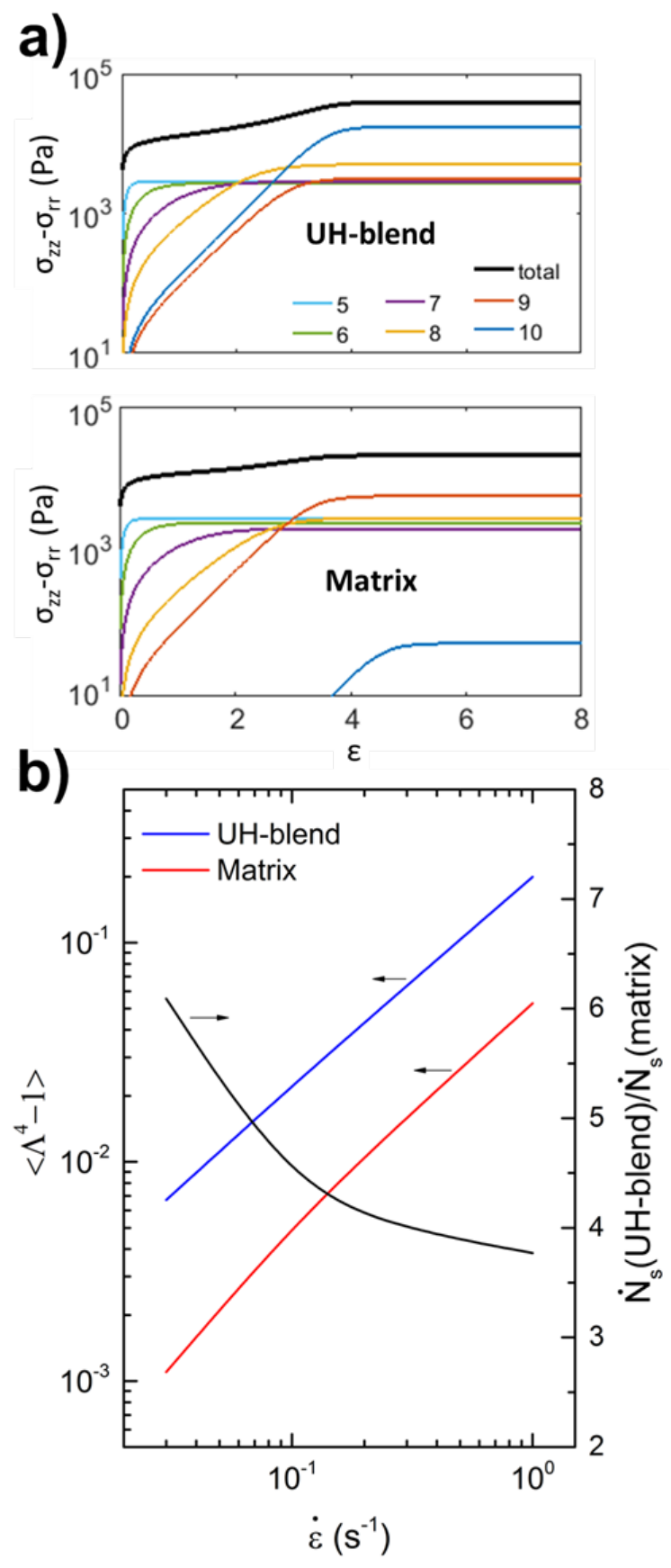

Figure 4: HMMSF analysis (a) Extensional stress. Coloured lines indicate the contribution from the different modes to the total stress (black line). Note the difference between the two samples for the longest mode (10th mode). (b) crystallization rate at the point of quench. Red and blue lines indicate average crystallization rate proportionality of the matrix and the UH-blend, respectively. The black line indicates the ratio of shish nucleation rate between UH-blend and matrix. 
tion rate proportionality $\left(\left\langle\Lambda^{4}-1\right\rangle\right)$ from the stretch $f_{i}$ of each mode in the HMMSF model weighted by $g_{i}$ :

$$
\left\langle\Lambda^{4}-1\right\rangle=\frac{1}{G_{N}^{0}} \sum_{i} g_{i}\left(\left\langle f_{i}^{4}\right\rangle-1\right)
$$

where $G_{N}^{0}=2.5 \mathrm{GPa}$ is the plateau modulus ${ }^{45}$ and

$$
\left\langle f_{i}^{4}\right\rangle=\frac{\int_{-\infty}^{t}\left(\partial G_{i}\left(t-t^{\prime}\right) / \partial t^{\prime}\right) f_{i}\left(t, t^{\prime}\right)^{4} d t^{\prime}}{\int_{-\infty}^{t}\left(\partial G_{i}\left(t-t^{\prime}\right) / \partial t^{\prime}\right) d t^{\prime}}
$$

Figure 4 shows the obtained values of $\left\langle\Lambda^{4}-1\right\rangle$ for both samples. At the highest Hencky strain rate investigated $\left(\dot{\varepsilon}=1 s^{-1}\right)$, the value of $\left\langle\Lambda^{4}-1\right\rangle$ for the UH-blend is a factor of 4 higher than that of the matrix. Hence, according to the model, the addition of $1 \mathrm{w} \%$ UHMwPE to the matrix, would result in an increase by a factor of 4 in $\dot{N}_{s}$.

To elucidate the connection between crystalline orientation and flow we plot in Figure 5 the final crystalline orientation $F_{H}$ versus steady stress. Although the data contain some uncertainty, $F_{H}$ for the two samples appear to collapse onto a single master curve. The experimental relationship between orientation and stress shows a power law dependency with a slope of $2 / 5$ rather than the linear relation reported for non-crystallizing systems. ${ }^{46}$ The apparent collapse of data onto one master curve is in accordance with previous findings for long chain branched polyethylene. ${ }^{31}$

We have shown that a very small fraction of UHMw-component in a semicrystalline polymer melt changes the extensional rheology as well as the final morphology significantly. The link between extensional rheology and enhanced formation of oriented structures lies in their mutual inherent sensitivity to chain stretch. The extensional response enables the evolution of the high molecular weight tail stretch to be studied in detail, up to steady flow. Thus we conclude that nonlinear extensional rheology particularly in connection with linear creep, provides a powerful tool for the study of threadlike precursors and shish formation in polymers of high polydispersity and/or with a UHMw-tail. 


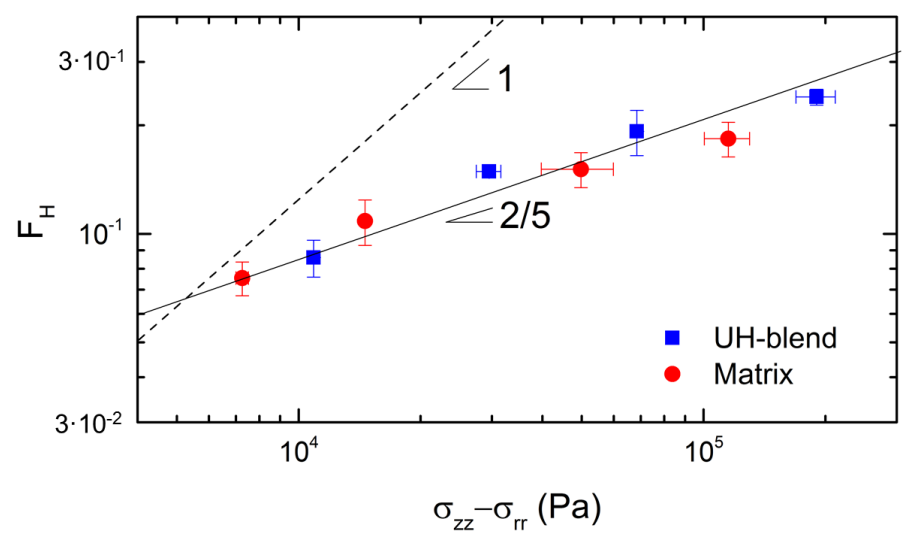

Figure 5: Herman's orientation factor versus steady stress for the UH-blend (blue) and matrix (red). Black solid and dashed line shows the apparent slope of $2 / 5$ and the expected trend for amorphous systems, respectively.

Materials and Methods Blending of UHMwPE and commercial HDPE was carried out in solution using hot xylene with subsequent precipitation in cold methanol according to procedures described elsewhere. ${ }^{47,48}$ The density of the commercial HDPE was $0.958 \mathrm{~g} / \mathrm{cm}^{3}$ which is high, hence the degree of branching was assumed to be very small.

SAOS was carried out in a strain controlled ARES G2 rheometer (TA instruments) and creep in a stress controlled rheometer MCR702 (Anton Paar, Austria). In both cases an 8 mm plate-plate geometry was used and the measurements were carried out in inert atmosphere $\left(N_{2}\right)$. For SAOS, we performed frequency sweeps at temperatures between $140-190{ }^{\circ} \mathrm{C}$. For creep, measurements were carried out at $150^{\circ} \mathrm{C}$ at different stresses (see Supporting Information for further experimental details and compliance curves). Compliance curves were subsequently inverted to obtain $G^{\prime}$ and $G^{\prime \prime}$ via multimode Maxwell fit using the software NLReg based on a generalized Tikhonov regularization. ${ }^{49}$ For each sample, SAOS and inverted creep data were shifted via time-temperature superposition principle and combined into one master curve at the reference temperature $T=140^{\circ} \mathrm{C}$.

We performed nonlinear extensional rheology using a Filament Stretch Rheometer (VADER 1000 from Rheofilament) comprising a moving top and a stationary bottom plate between which a sample disc was placed. Uniaxial extension at a constant Hencky strain rate was performed at $140{ }^{\circ} \mathrm{C}$ by axial movement of the top plate. The deformation of the midfil- 
ament plane, monitored via a laser micrometer, was measured in terms of Hencky strain

$\varepsilon=-2 \ln \frac{D(t)}{D_{0}}$ where $D(t)$ and $D_{0}$ are the measured and the initial diameter, respectively. A feedback control loop enabled controlled deformation and prevented failure due to uncontrolled neck propagation. ${ }^{50-53}$ The normal stress difference in the material is given by $\left\langle\sigma_{z z}-\sigma_{r r}\right\rangle=\left(F-\frac{1}{2} m g\right) / \frac{\pi}{4} D(t)^{2}$. Here $F$ is the force measured on the bottom plate, $m$ is the mass of the sample and $g$ is the gravitational acceleration. The filaments were quenched to room temperature at $\varepsilon=5.5$ at a rate $>10 \mathrm{~K} / \mathrm{s}$. The diameter at quench for all samples were $0.47-0.5$. (see Supporting Information for further details on extension)

Ex-situ SAXS patterns from the midfilament plane of quenched samples were collected using a SAXSLAB instrument (Ganesha from SAXSLAB, Denmark) with a 300k Pilatus detector (pixel sizes $172 \times 172 \mu \mathrm{m}$ ). The wavelength of the X-ray beam was $1.54 \AA$ and the sample-to-detector distance was $1491 \mathrm{~mm}$. Exposure times were $10-170 \mathrm{~min}$.

\section{Acknowledgement}

The authors thank Aage og Johanne Louis-Hansen fonden for financial support. Furthermore we thank Qian Huang and Prof. Nicolas J. Alvarez for fruitful discussions on the work.

\section{Supporting Information Available}

The following files are available free of charge.

- SupportingInformation.pdf: Figures S1 - S5 and, Table S1 and supplemental text

\section{References}

(1) van Erp, T. B.; Balzano, L.; Spoelstra, A. B.; Govaert, L. E.; Peters, G. W. M. Quantification of non-isothermal, multi-phase crystallization of isotactic polypropylene: The influence of shear and pressure. Polymer 2012, $\underline{53}, 5896$. 
(2) van Drongelen, M.; Cavallo, D.; Balzano, L.; Portale, G.; Vittorias, I.; Bras, W.; Alfonso, G. C.; Peters, G. W. M. Structure Development of Low-Density Polyethylenes During Film Blowing: A Real-Time Wide-Angle X-ray Diffraction Study. Macromol. Mater. Eng. 2014, 299, 1494-1512.

(3) Graham, R. S.; Olmsted, P. D. Coarse-Grained Simulations of Flow-Induced Nucleation in Semicrystalline Polymers. Phys. Rev. Lett. 2009, 103, 1-4.

(4) Meijer, H. E. H. Materials Science and Technology, Processing of Polymers, 11th ed.; Materials Science and Technology; Wiley, 1997; p 787.

(5) Pennings, A. J.; van der Hooft, R. J.; Postema, A. R.; Hoogsteen, W.; ten Brinke, G. High-speed gel-spinning of ultra-high molecular weight polyethylene. Polymer Bulletin 1986, 16, 167-174.

(6) Pennings, A. Further studies on the high-speed gel-spinning of ultra-high molecular weight polyethylene. Polymer Bulletin 1990, 23.

(7) Schrauwen, B. A. G.; Breemen, L. C. A. V.; Spoelstra, A. B.; Govaert, L. E.; Peters, G. W. M.; Meijer, H. E. H. Structure, deformation, and failure of flow-oriented semicrystalline polymers. Macromolecules 2004, 37, 8618.

(8) Keller, A.; Kolnaar, H. W. H. Materials science and technology; VCH Verlagsgesellschaft mbH, 1997; pp $187-268$.

(9) Marrucci, G. Fast flows of concentrated polymers - predictions of the tube model on chain stretching. Gazzetta Chimica Italiana 1988, 118.

(10) Graham, R. S.; Likhtman, A. E.; McLeish, T. C. B.; Milner, S. T. Microscopic theory of linear, entangled polymer chains under rapid deformation including chain stretch and convective constraint release. Journal of Rheology 2003, 47, 1171-1200. 
(11) Janeschitz-Kriegl, H. Crystallization Modalities in Polymer Melt Processing: Fundamental Aspects of Structure Formation; Springer Vienna: Vienna, 2010.

(12) Shen, B.; Liang, Y.; Kornfield, J. A.; Han, C. C. Mechanism for Shish Formation under Shear Flow: An Interpretation from an in Situ Morphological Study. Macromolecules 2013, $\underline{46}, 1528-1542$.

(13) Yan, T.; Zhao, B.; Cong, Y.; Fang, Y.; Cheng, S.; Li, L.; Pan, G.; Wang, Z.; Li, X.; Bian, F. Critical Strain for Shish-Kebab Formation. Macromolecules 2010, 43, 602-605.

(14) Seki, M.; Thurman, D. W.; Oberhauser, J. P.; Kornfield, J. A. Shear-mediated crystallization of isotactic polypropylene: The role of long chain-long chain overlap. Macromolecules 2002, 35, 2583-2594.

(15) Hsiao, B. S.; Yang, L.; Somani, R. H.; Avila-Orta, C. A.; Zhu, L. Unexpected ShishKebab Structure in a Sheared Polyethylene Melt. Phys. Rev. Lett. 2005, 94, 117802.

(16) Kimata, S.; Sakurai, T.; Nozue, Y.; Kasahara, T.; Yamaguchi, N.; Karino, T.; Shibayama, M.; Kornfield, J. A. Molecular Basis of the Shish-Kebab Morphology in Polymer Crystallization. Science 2007, 316, 1014-1017.

(17) Yang, L.; Somani, R. H.; Sics, I.; Hsiao, B. S.; Kolb, R.; Lohse, D. The role of high molecular weight chains in flow-induced crystallization precursor structures. Journal of Physics: Condensed Matter 2006, 18, S2421-S2436.

(18) Nogales, A.; Hsiao, B.; Somani, R.; Srinivas, S.; Tsou, A.; Balta-Calleja, F.; Ezquerra, T. Shear-induced crystallization of isotactic polypropylene with different molecular weight distributions: in situ small- and wide-angle X-ray scattering studies.

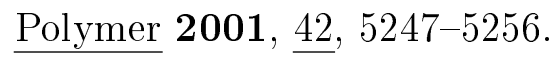

(19) Balzano, L.; Kukalyekar, N.; Rastogi, S.; Peters, G. W. M.; Chadwick, J. C. Crystal- 
lization and Dissolution of Flow-Induced Precursors. Physical Review Letters 2008, $\underline{100,} 048302$.

(20) Ogino, Y.; Fukushima, H.; Matsuba, G.; Takahashi, N.; Nishida, K.; Kanaya, T. Effects of high molecular weight component on crystallization of polyethylene under shear flow. Polymer 2006, 47, 5669-5677.

(21) Matsuba, G.; Sakamoto, S.; Ogino, Y.; Nishida, K.; Kanaya, T. Crystallization of Polyethylene Blends under Shear Flow. Effects of Crystallization Temperature and Ultrahigh Molecular Weight Component. Macromolecules 2007, 40, 7270-7275.

(22) Bird, R. $\quad$ B.; Armstrong, R. $\quad$ C.; Hassanger, O. Dynamics of polymeric liquids. Vol.1: fluid mechanics; Wiley, 1987.

(23) Costanzo, S.; Huang, Q.; Ianniruberto, G.; Marrucci, G.; Hassager, O.; Vlassopoulos, D. Shear and Extensional Rheology of Polystyrene Melts and Solutions with the Same Number of Entanglements. Macromolecules 2016, 49, 3925-3935.

(24) Cui, K.; Meng, L.; Tian, N.; Zhou, W.; Liu, Y.; Wang, Z.; He, J.; Li, L. Self-Acceleration of Nucleation and Formation of Shish in Extension-Induced Crystallization with Strain Beyond Fracture. Macromolecules 2012, 45, 5477-5486.

(25) Cui, K.; Ma, Z.; Wang, Z.; Ji, Y.; Liu, D.; Huang, N.; Chen, L.; Zhang, W.; Li, L. Kinetic Process of Shish Formation: From Stretched Network to Stabilized Nuclei. Macromolecules 2015, 48, 5276-5285.

(26) Derakhshandeh, M.; Hatzikiriakos, S. G. Flow-induced crystallization of high-density polyethylene: The effects of shear and uniaxial extension. Rheologica Acta 2012, 51, $315-327$.

(27) Wang, Z.; Ju, J.; Yang, J.; Ma, Z.; Liu, D.; Cui, K.; Yang, H.; Chang, J.; Huang, N.; 
Li, L. The non-equilibrium phase diagrams of flow-induced crystallization and melting of polyethylene. Sci. Rep. 2016, $\underline{6}, 32968$.

(28) Sentmanat, M.; Wang, B. N.; McKinley, G. H. Measuring the transient extensional rheology of polyethylene melts using the SER universal testing platform. Journal of

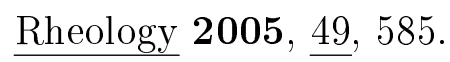

(29) White, E. E. B.; Winter, H. H.; Rothstein, J. P. Extensional-flow-induced crystallization of isotactic polypropylene. Rheologica Acta 2012, 51, 303.

(30) Hadinata, C.; Boos, D.; Gabriel, C.; Wassner, E.; Rüllmann, M.; Kao, N.; Laun, M. Elongation-induced crystallization of a high molecular weight isotactic polybutene-1 melt compared to shear-induced crystallization. Journal of Rheology 2007, 51.

(31) Wingstrand, S. L.; van Drongelen, M.; Mortensen, K.; Graham, R. S.; Huang, Q.; Hassager, O. Influence of Extensional Stress Overshoot on Crystallization of LDPE. Macromolecules 2017, 50, 1134-1140.

(32) Gabriel, C.; Kaschta, J.; Münstedt, H. Influence of molecular structure on rheological properties of polyethylenes. Rheologica Acta 1998, 37, 7-20.

(33) Münstedt, H.; Schwarzl, F. R. Deformation and flow of polymeric materials; Springer Berlin Heidelberg, 2014; p 558.

(34) Alvarez, N. J.; Marín, J. M. R.; Huang, Q.; Michelsen, M. L.; Hassager, O. Creep Measurements Confirm Steady Flow after Stress Maximum in Extension of Branched Polymer Melts. Physical Review Letters 2013, 110, 168301.

(35) Narimissa, E.; Wagner, M. H. A hierarchical multimode molecular stress function model for linear polymer melts in extensional flows. Journal of Rheology 2016, 60, 625-636.

(36) Wagner, M. H.; Bastian, H.; Hachmann, P.; Meissner, J.; Kurzbeck, S.; Münstedt, H.; 
Langouche, F. The strain-hardening behaviour of linear and long-chain-branched polyolefin melts in extensional flows. Rheologica Acta 2000, 39, 97-109.

(37) Doi, M.; Edwards, S. The theory of polymer dynamics; Clarendon, 1986; p 391.

(38) Currie, P. Constitutive equations for polymer melts predicted by the Doi-Edwards and Curtiss-Bird kinetic theory models. Journal of Non-Newtonian Fluid Mechanics 1982, $\underline{11}, 53-68$.

(39) Hassager, O.; Hansen, R. Constitutive equations for the Doi-Edwards model without independent alignment. Rheologica Acta 2010, 49, 555-562.

(40) Urakawa, O.; Takahashi, M.; Masuda, T.; Ebrahimi, N. G. Damping Functions and Chain Relaxation in Uniaxial and Biaxial Extensions: Comparison with the DoiEdwards Theory. Macromolecules 1995, 28, 7196-7201.

(41) Zuidema, H.; Peters, G. W. M.; Meijer, H. E. H. Development and Validation of a Recoverable Strain-Based Model for Flow-Induced Crystallization of Polymers. Macromolecular Theory and Simulations 2001, 10, 447-460.

(42) Roozemond, P. C.; Peters, G. W. M. Flow-enhanced nucleation of poly(1-butene): Model application to short-term and continuous shear and extensional flow. Journal of Rheology 2013, 57, 1633-1653.

(43) Steenbakkers, R. J. A.; Peters, G. W. M.; Meijer, H. E. H. Rheological modeling of flowinduced crystallization in polymer melts and limitations on classification of experiments. AIP Conference Proceedings 2008, 1027, 493-495.

(44) Likhtman, A. E.; Graham, R. S. Simple constitutive equation for linear polymer melts derived from molecular theory: RolieâĂŞPoly equation. Journal of Non-Newtonian Fluid Mechanics 2003, 114, 1-12. 
(45) Fetters, L. J.; Lohse, D. J.; Colby, R. H. In Physical Properties of Polymers; 2, Ed.; 2007; p 1073.

(46) López-Barrón, C. R.; Zeng, Y.; Richards, J. J. Chain stretching and recoiling during startup and cessation of extensional flow of bidisperse polystyrene blends. Journal of Rheology 2017, 61, 697-710.

(47) Diop, M. F.; Burghardt, W. R.; Torkelson, J. M. Well-mixed blends of HDPE and ultrahigh molecular weight polyethylene with major improvements in impact strength achieved via solid-state shear pulverization. Polymer (United Kingdom) 2014, $\underline{55}, 4948$ 4958.

(48) Yang, L.; Somani, R. H.; Sics, I.; Hsiao, B. S.; Kolb, R.; Fruitwala, H.; Ong, C. ShearInduced Crystallization Precursor Studies in Model Polyethylene Blends by in-Situ Rheo-SAXS and Rheo-WAXD. Macromolecules 2004, 37, 4845-4859.

(49) Honerkamp, J.; Weese, J. A nonlinear regularization method for the calculation of

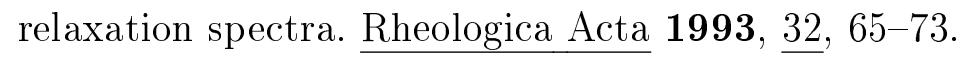

(50) Román Marín, J. M.; Huusom, J. K.; Alvarez, N. J.; Huang, Q.; Rasmussen, H. K.; Bach, A.; Skov, A. L.; Hassager, O. A control scheme for filament stretching rheometers with application to polymer melts. Journal of Non-Newtonian Fluid Mechanics 2013, $\underline{194}, 14$.

(51) Fielding, S. M. Criterion for Extensional Necking Instability in Polymeric Fluids. Physical Review Letters 2011, 107, 258301.

(52) Hoyle, D. M.; Fielding, S. M. Criteria for extensional necking instability in complex fluids and soft solids. Part I: Imposed Hencky strain rate protocol. Journal of Rheology 2016, $\underline{60}, 1347-1375$. 
(53) Hoyle, D. M.; Fielding, S. M. Criteria for extensional necking instability in complex fluids and soft solids. Part II: Imposed tensile stress and force protocols. Journal of

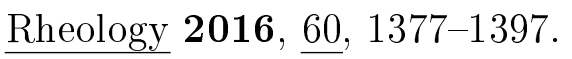




\section{Graphical TOC Entry}

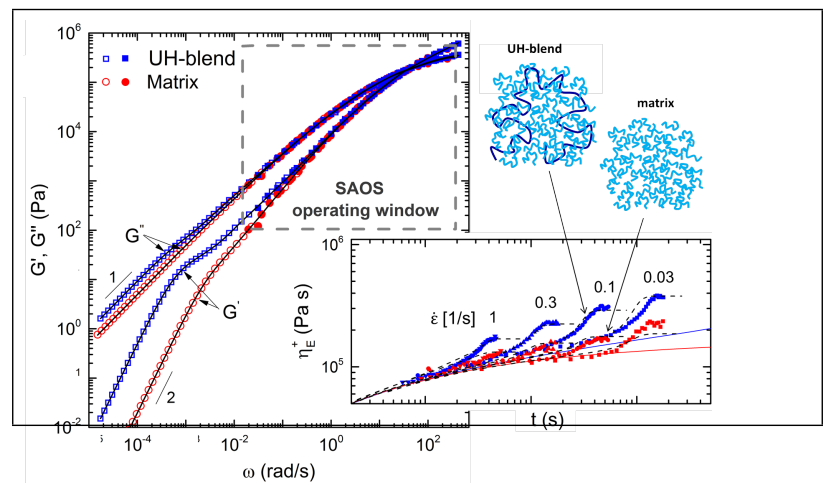

